\title{
Dementia
}

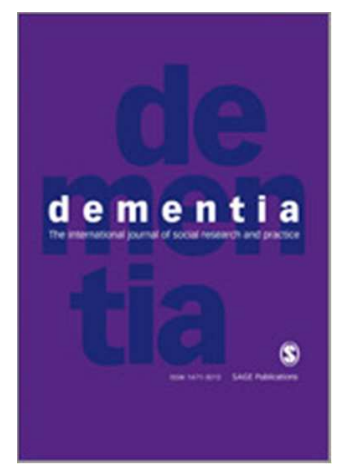

\section{Improving access to a multi-component intervention for caregivers and people with dementia}

\begin{tabular}{|r|l|}
\hline Journal: & Dementia: the international journal of social research and practice \\
\hline Manuscript ID & Draft \\
\hline Origin of Contribution: & UK/Europe and the rest of the world \\
\hline Manuscript Type: & Original Article \\
\hline Keywords: & $\begin{array}{l}\text { dementia, informal caregivers, multicomponent intervention, costs, } \\
\text { improving access }\end{array}$ \\
\hline Abstract: & $\begin{array}{l}\text { Due to the increasing social and economic costs of dementia, there are } \\
\text { urgent calls to develop accessible and sustainable care for people with } \\
\text { dementia and their caregivers. Multi-component non-pharmacological } \\
\text { interventions (NPIs) appear effective in improving or maintaining daily } \\
\text { functioning and well-being, but are typically labour-intensive for health } \\
\text { care professionals, thus hindering access. The current study aimed to } \\
\text { explore the feasibility and acceptability of a novel approach to widen } \\
\text { access to NPI by involving caregivers to present part of the intervention } \\
\text { and with staff from local support organizations instructed to train the } \\
\text { caregivers. Trainers and caregivers were shown to comply with training } \\
\text { instructions and the direct intervention costs were low. Feedback from } \\
\text { trainers and caregivers was positive and well-being ratings from people } \\
\text { with dementia and caregivers remained stable over time and caregivers' } \\
\text { sense of competence improved. The findings suggest that involving } \\
\text { caregivers and trained non-professionals to provide the intervention is } \\
\text { feasible and acceptable and could be a cost-effective solution to improve } \\
\text { access to care. }\end{array}$ \\
\hline
\end{tabular}

\section{SCHOLARONE \\ Manuscripts}




\section{Dementia}

\section{Introduction}

Because of the increasing social and economic costs of dementia, the urgency of developing cost-effective care for people with dementia and their caregivers has been emphasized (Wimo \& Prince, 2010). Non-pharmacological interventions (NPIs) are recognised as valuable alternatives or complements to medication, and for caregivers of people with dementia NPIs are typically the only option (Jensen, Agbata, Canavan \& McCarthy, 2014; Chien et al., 2011). Multi-component NPIs combine therapeutic strategies directed at the person with dementia and their informal caregiver (Eloniemi-Sulkava et al., 2009; Mittelman, Roth, Coon, \& Haley, 2004; Prick, de Lange, Twisk \& Pot, 2015). Multi-component NPIs appear particularly effective in maintaining well-being and daily functioning in the person with dementia, and improving caregiver well-being (Van 't Leven et al., 2013; Olazarán et al., 2010), which in turn can have positive cost implications. Caregiver well-being and burden, and daily functioning in the person with dementia, are reliable predictors of health service use and nursing home admission (Gaugler, Yu, Krichbaum, \& Wyman, 2009; Robinson et al., 2010). Therefore, improving well-being, burden and daily functioning can postpone nursing home admission resulting in reductions in health care costs (Olazarán et al., 2010). Despite evidence for the effectiveness of multicomponent NPIs, there have been few attempts to implement such interventions into support services (Gitlin, Marx, Stanley, \& Hodgson, 2015) and specialised services to provide NPIs for people with dementia and caregivers are still in short supply, especially in rural areas (Wimo \& Prince, 2010; Robinson et al, 2010).

A disadvantage of most current multi-component NPIs is their high cost, as the interventions typically are labour-intensive and rely on highly trained health care professionals (e.g. nurses, occupational therapists). High costs could make multicomponent NPIs less attractive options for sustainable, widely accessible long-term care and could be a reason for 


\section{Dementia}

their limited implementation. Important factors contributing to intervention costs are the time required for training professional staff to present the intervention and the staff time required to actually deliver the intervention (Eloniemi-Sulkava et al., 2009; Graff et al., 2008). Additional factors relevant for intervention implementation include the efficiency of the staff training, i.e. can staff present the intervention as intended, and the demands that presenting the intervention puts on health care services (NHS Education for Scotland, 2011).

The aim of the current study was to pilot a novel approach of presenting multicomponent NPIs that could be more sustainable and improve access to the intervention. To reduce staff costs and demands on health services, this approach involved informal caregivers of people with dementia to present part of the intervention and staff from local charities and support organizations were recruited to train the caregivers. In turn, these trainers were instructed by a health care professional. This cascade model of training was expected to improve access to the intervention without greatly increasing the workload of health care professionals and the associated health care costs. Training manuals limited the time required for training staff and caregivers and helped to promote treatment fidelity (Bellg et al., 2004). Previous studies have shown that training family caregivers to present NPIs is feasible and acceptable for both people with dementia and caregivers (Gitlin et al., 2009; Milders, Bell, Lorimer, MacEwan, \& McBain, 2013; Quayhagen \& Quayhagen, 2001; Teri et al., 2003). However, involving staff from local charities and voluntary organisations to train caregivers has not been explored extensively. Therefore, before the effectiveness of intervention can be evaluated, the main questions in the current study were whether this approach of presenting a NPI was feasible and acceptable. Important criteria for feasibility were treatment integrity, low costs and limited time investment for health care professionals. Criteria to evaluate acceptability were being valued by recipients, confidence in trainers and caregivers and no adverse impact on well-being or quality-of life in the caregiver or person with dementia. These 
questions of feasibility and acceptability were examined in two studies. The focus in Study 1 was treatment fidelity, costs and acceptability of the intervention and in Study 2 the feasibility of implementing the intervention in the community.

\section{Study 1}

\section{Method}

\section{Design.}

The study followed a cross-over design. Participants were allocated to a treatment group or a waiting list (followed by delayed treatment) group. Both groups were assessed on three occasions; at baseline, 3 months and 6 months after baseline. The treatment group received the intervention between the baseline and 3-month assessments. The waiting list group received the intervention between the 3-month and 6-month assessment. The study had been approved by the XXX Research Ethics Committee (REC reference number XXX).

\section{Participants.}

Thirty dyads, consisting of a person with dementia and their main informal caregiver gave informed consent to take part in this study. The participants were recruited via the care team of the person with dementia, the database of the XXX Dementia Clinical Research Network or locally through health and social care organisations. Main exclusion criteria for both the person with dementia and the caregiver were: participation in another study; major mental illness (e.g. psychosis, depression) or major physical impairments (e.g. severe cardiac or respiratory illness); inability to give informed consent. Type of dementia was not a selection criterion. The research coordinator (author XXX), a mental health nurse with extensive 
experience of dementia care and training in psychosocial methods of care, met all the participants to check for exclusion criteria and take informed consent. Observational assessment of dementia severity by the research coordinator indicated mild to moderate severity in most participants with dementia. Age and gender distribution in the sample are displayed in Table 1. The relationship of the caregivers to the person with dementia were: wife (18), husband, (4), daughter (7) or daughter-in-law (1). The caregivers either lived with the person with dementia or visited the person with dementia at least three times per week.

Dyads were allocated to an immediate treatment group or a waiting list group in a pseudo-random order. The allocation process aimed to ensure that all caregivers could receive the training during the course of the study at the centre nearest to their home. Sixteen dyads were allocated to the treatment group and 14 to the waiting-list group. Mean age of the persons with dementia and of the caregivers in the two groups were comparable $(t(28)=1.90$, $p>.06$ and $t(28)=0.62, p>.54$, respectively, see Table 1$)$. The treatment group contained more men than women with dementia (Chi square $=6.25, p=.012$ ); in the waiting list group the gender distribution was not different ( $p=.59$, see Table 1$)$. Both groups had more female caregivers than male caregivers (Chi square $>=7.14, p<.01$ ). All 30 dyads completed the baseline and 3-month assessments. Twenty-three dyads (12 treatment, 11 waiting list) completed the 6-month follow-up. Of those dyads who did not complete the 6-month followup, three dyads had started too late to complete the third assessment and four dyads dropped out; one person with dementia went into residential care, one died and two dyads withdrew for family reasons.

Table 1 about here

Trainers. 
Fourteen trainers were recruited from charities and support organisations in XXX. All trainers had experience of dementia care, but varied in their levels of education and experience. Only three trainers were qualified health care professionals. Educational level of the remaining trainers ranged from secondary school only to university level. All trainers were instructed by the research coordinator. Trainers were also provided with a trainers' manual containing step-by-step instructions on how to deliver the caregiver training. The instructions emphasised a person-centred approach, to individualise activities to suit the interests, experience and abilities of the person with dementia and caregiver. Trainers were instructed in a single session lasting approximately two hours. In total there were seven training sessions with one to four trainers per session.

\section{Intervention.}

The manual-based intervention comprised several components: educating caregivers on dementia and living with dementia, training caregivers in effective communication, coping with stress and training caregivers to stimulate the person with dementia both physically and mentally through activities. The manual for caregivers presented the above components and contained detailed descriptions of activities that the caregiver could do at home with the person with dementia, including reminiscence activities, household tasks, quizzes and light physical exercises. Several activities have previously been used in cognitive stimulation therapy (CST), which has shown to have a positive effect on quality of life in the person with dementia (Aguirre, Woods, Spector \& Orrell, 2013). Furthermore, physical activities have shown to be beneficial for well-being and mood in people with dementia (Teri et al., 2003; Van ' $t$ Leven et al., 2013). The manual also contained resource materials that could be used during the activities, e.g. crosswords, photographs. The intervention and manual had been developed 


\section{Dementia}

in consultation with health care professionals, people with dementia, caregivers and representatives of dementia organisations.

The caregiver training addressed the components of the intervention and caregivers were shown how to use the manual and how to engage the person with dementia in stimulating activities. The importance of a person-centred approach was emphasised, including tailoring activities to suit the individual and choosing activities that the person with dementia enjoyed. Caregiver training took place in eight small groups of two to four caregivers, led by two trainers, over four weekly sessions lasting approximately 90 minutes each. Five caregivers received individual training as no training group was available at the time. After two training sessions caregivers were encouraged to start engaging their relative with dementia in activities at home, three times per week. During the last two sessions caregivers had the opportunity to discuss progress or difficulties implementing the activities at home.

\section{Assessments.}

Assessment measures were chosen to assess criteria for feasibility (treatment fidelity, costs) and acceptability (no negative impact on wellbeing, positive feedback from recipients).

Treatment fidelity. To assess whether the trainers trained the caregivers in accordance with instructions, the research coordinator observed trainers delivering the caregiver training and rated their performance against eight predetermined criteria as good, adequate or less than adequate. To assess whether the caregivers presented the activities to the person with dementia as intended, audio-recordings were made of dyads carrying out an activity at home, with their consent. The recordings were scored against a list of 22 criteria that should be present (e.g. caregiver offers person with dementia choice of activity) or absent (e.g. distracting background noise) when presenting the activity. Caregivers were asked to indicate in the manual the activities they had carried out with their relative with dementia, how many 
times a week they had engaged in activities and a subjective assessment of how much their relative had enjoyed the activity, ranging between not enjoyed (1) and enjoyed a lot (4).

Costs. The costs of the intervention were estimated from the resources required to deliver the training, as recorded by the research coordinator. The impact of the intervention on health and social care service costs was estimated based on reported service use in the person with dementia and caregiver in the last 30 days, as completed by the caregiver, using the RUD-Lite (Wimo, Karlsson, Jönsson, \& Winblad, 1998), which is a frequently used instrument to assess resource use in dementia. The costs of care use and time input from health care staff and other trainers were estimated using published standard unit costs in the United Kingdom (Curtis, 2013).

Well-being and daily functioning. Quality-of-life and daily functioning in the person with dementia was assessed with the Quality of Life-Alzheimer's Disease scale (QoL-AD; Logdon, Gibbons, McCurry \& Teri, 1999), a self-report questionnaire with good reliability and validity, designed to allow people with severe dementia to complete the rating, and the Physical Self-Maintenance/ Instrumental Activities of Daily Living (PSMS/IADL; Lawton \& Brody, 1969), a scale for assessment of daily functioning of the person with dementia, as recorded by the caregiver.

Quality-of-life in the caregiver was assessed using the Short-Form Health Survey (SF12; Ware, Kosinski, \& Keller, 1996), a widely used self-report quality-of-life measure. Caregiver burden was assessed with the self-report Zarit Burden Interview (ZBI; Zarit, Orr, \& Zarit, 1985).

Feedback._When the caregiver training was completed, trainers were asked to evaluate the training that they had received in a 17 -item questionnaire, to be returned anonymously. At the 6-month assessment caregivers received a 17 -item questionnaire to evaluate the manual and the training that they had received. The questionnaire could be completed at home and returned anonymously. 


\section{Statistical analysis.}

The ratings of observations and recordings to assess treatment fidelity were summarised in descriptive statistics as were the feedback ratings from trainers and caregivers. Estimated health care and social care costs were compared between the treatment and waiting list groups at the 3-month and 6-month assessments in between group comparisons using non-parametric Mann-Whitney tests. Similarly, ratings on the well-being measures for caregivers and people with dementia and daily functioning of the person with dementia were compared between the treatment and waiting list group at the three assessment points using non-parametric tests.

\section{Results}

\section{Treatment fidelity.}

Trainers. Of the eight caregiver groups that the trainers instructed, each was observed once by the research coordinator. A session was scored on eight criteria, resulting in a total of 64 ratings across all groups. Only three ratings were less than adequate, six were adequate and $55(86 \%)$ were good. No training session received more than one "less than adequate" rating and five out of eight sessions were rated as "good" throughout.

Caregivers. Ten dyads gave permission to record a session at home. The recordings were rated by two independent raters (authors XXX and XXX, both experienced clinical psychologists); interrater reliability was high ( $r=.72)$. Overall adherence to the main features of the training was good. On average $83.2 \%$ of the criteria were endorsed, meaning that behaviour that had been recommended during the caregiver training was observed and behaviour that had been discouraged was not observed. The raters gave an overall judgement 
of each session ranging from good (1) to unsatisfactory (4). On average the sessions were deemed adequate to good $(M=1.8 \mathrm{SD}=0.58)$. Only one session was rated as less than adequate.

Table 2 about here

\section{Health service resource use and costs.}

Estimated health care and social care costs were compared between the treatment and the waiting list groups at 3-month and 6-month follow-up. Total health service costs for caregivers and persons with dementia did not differ between the groups ( $p>.10$, see Table 2). One cost category (visits to general practitioners, other specialists or social workers) was significantly higher in persons with dementia in the intervention group (M 1156.88, SD 2134.79) than in the waiting-list group ( $M$ 314.93, SD 598.47) at 3-month follow-up ( $Z=1.43$, $p<.05)$

The direct costs of the intervention were based on the time that trainers spent on receiving training and presenting the caregiver training using standard unit costs (Curtis 2013), taking into account grade and salary level. Additional costs included were venue hire, travel costs for the purpose of training and printing costs for the manuals. Costs of the research coordinator were not included as these were incurred for research purposes only. The total cost of the intervention was estimated at $£ 2,258$, meaning an average intervention cost per dyad of $£ 75$ ( $€ 104$ or USD 115).

\section{Wellbeing and daily functioning.}




\section{Dementia}

Ratings on the SF-12 were transformed into SF-6D scores to ease interpretation and those scores are reported here. The SF-6D provides a single index of health related quality of life, ranging from 0 (worst) to 1 (best). Comparing SF-6D and ZBI scores between groups at baseline, 3-month and 6-month assessment revealed no significant group differences ( $p>=.2$ ). Comparing QoL-AD and PSMS/IADL ratings for the person with dementia between the two groups at the three assessments also failed to reveal significant differences ( $p>=.06)$. Caregivers and people with dementia in the two groups did not differ prior to the intervention and maintained baseline levels on well-being, daily functioning and burden.

\section{Feedback.}

Trainers. Thirteen of the 14 trainers returned the questionnaire evaluating the training. Overall trainers were very positive about the manuals and the training that they had received, indicating that the instructions were clear (13/13) and that they had understood the material (13/13); most also indicated that the training was useful for their area of work and had increased their confidence to train caregivers (12/13). According to most trainers, the caregivers in their group had understood the instructions $(12 / 13)$ and had benefitted from the training $(11 / 13)$.

Caregivers. Twenty-seven caregiver manuals were returned at the end of the study. The number of activities recorded in the manuals varied widely. Seven caregivers recorded less than 10 activities and other caregivers indicated "does daily" for an activity. Therefore, it is difficult to estimate the total number of activities that dyads carried out during the study. What is clear is that most activities presented were enjoyed by the person with dementia; $77 \%$ of the activities were rated as "enjoyed a lot" and $20 \%$ as "quite enjoyed". Activities that were enjoyed best were presented more frequently. Popular activities were household tasks (e.g. 
drying dishes), current affairs, musical reminiscence, "life story work" and walking and stair walking exercises.

Nineteen out of 30 caregivers returned the questionnaire evaluating the training they had received. Seventeen (89\%) found the manual useful, 15 (79\%) found the training useful and all 19 indicated that the material had been presented clearly by the trainers. Sixteen (84\%) caregivers indicated that their relative with dementia had enjoyed the activities and 12 (63\%) indicated that their relative with dementia had benefitted from the activities, but two caregivers reported negative effects in their relative with dementia, such as increased irritation or confusion. Sixteen (84\%) caregivers reported that doing the activities with their relative with dementia was never or rarely a burden for them and $12(63 \%)$ thought that they themselves had benefitted from doing the activities with their relative. Sixteen (84\%) caregivers intended to continue with the activities after the study had ended.

\section{Study 2}

\section{Method}

\section{Design.}

The study had a single condition repeated measure design. All participating caregivers received the training and were assessed at baseline, prior to training start, and three months later. The study had been approved by the XXX Research Ethics Committee (REC reference $X X X)$.

\section{Participants.}

Fifty-four informal caregivers of home-living people with dementia gave informed consent to take part and completed the baseline assessment. Caregivers were invited by the 


\section{Dementia}

trainers from the participating organisations. Mean age of the caregivers was 62.57 (SD 12.76), range 31-86 years. Five caregivers were male and 49 female. The relationships of the caregivers to the person with dementia were wife (20), daughter (23), daughter in law (3), granddaughter (1), husband (5), sister (1) or friend (1). Caregiver training took place in 14 groups consisting of 3-7 carers, over four weeks led by one or two trainers. Exclusion criteria and content of the caregiver training and manual were the same as in Study 1. Fifty caregivers completed and returned the outcome measures at the 3-month follow-up.

Trainers.

Thirty-six trainers were recruited from six different organisations in XXX. Four were dementia charities and voluntary organisations, one was a social care organisation and one was a health care organisation. All trainers had experience of dementia care, but varied in their levels of education and experience. Five trainers were qualified health care professionals. Educational level of the remaining trainers ranged from secondary school only to university level. The research coordinator instructed all trainers in groups of 3-6 staff. In more remote areas staff training could be on 1:1 basis. Training manual and instructions were the same as in Study 1.

\section{Assessments.}

Assessment of treatment fidelity in the trainers and of trainer and caregiver feedback of their training was the same as in Study 1, as were the measures of caregiver wellbeing (SF12, ZBI). An additional measure was the Caregiver Competence Questionnaire, a 7-item questionnaire to assess caregivers' feeling of competence to care for the person with dementia (Vernooij-Dassen et al., 1999). In addition to caregiver wellbeing, improving caregivers' sense of competence can also contribute to postponing nursing home admission of 
the person with dementia (Dröes, Breebaart, Meiland, van Tilburg \& Mellenbergh, 2004). The persons with dementia were not assessed in Study 2.

\section{Statistical analysis.}

The ratings of observations and recordings to assess treatment fidelity, as well as feedback ratings from trainers and caregivers were summarised in descriptive statistics. Caregiver wellbeing and confidence measures at baseline and follow-up were compared with non-parametric Wilcoxon tests.

Results

\section{Treatment fidelity.}

Eight sessions of caregiver training provided by the trainers were either observed (5) or recorded and scored afterwards (3) based on the same criteria as used in Study 1. All sessions were rated as adequate or good and three were rated as good throughout.

\section{Wellbeing and sense of competence.}

As in study 1, SF-12 scores were recoded into SF6D scores (see Table 3). Comparing ratings from before and after training using non-parametric Wilcoxon tests, showed no changes over time for caregiver burden or quality-of-life $(p>=.5)$, but did show a significant improvement in sense of competence $(Z=2.13, p<0.05)$. Caregivers felt more competent after the training.

Table 3 about here 


\section{Dementia}

\section{Feedback.}

Trainers._Of the 36 individuals the different organisations trained, 22 went on to train caregivers. Twenty of these 22 returned the questionnaire evaluating the training. All 20 indicated that the manual was clear and that they had understood the material. Seventeen (85\%) found the training useful and that it had increased their confidence to train caregivers.

Caregivers. Forty-six caregivers returned the questionnaire evaluating their training. All 46 indicated that they found the caregiver manual useful and that the trainers had presented the training material clearly, and 38 (82\%) found the training useful. All 46 caregivers indicated that doing the activities was never or rarely a burden for them and 37 (80\%) intended to continue using aspects of the training after the study had ended. Forty (87\%) caregivers found that their relative with dementia had benefitted from the activities, but six $(13 \%)$ indicated a negative effect on the person with dementia. Thirty-eight (82\%) caregivers indicated that they themselves had benefitted from doing the activities with their relative with dementia.

\section{Discussion}

In two studies we explored the feasibility and acceptability of involving caregivers to present parts of a multicomponent intervention and of instructing individuals working in dementia care, who were not health care professionals, to train the caregivers. This approach aimed to reduce costs of the intervention and improve access to the intervention without greatly increasing the burden on health care services. Of the six organisations involved in the two studies, only one was a health care organisation and only 8 of the 51 trainers across the two studies were fully qualified health care professionals. 
Results of both studies suggest that the approach is feasible and acceptable.

Treatment fidelity was satisfactory. Trainers followed the instructions on how to train the caregivers and most caregivers adhered to the instructions from the caregiver training. Notes in the caregiver manuals also indicated that caregivers chose activities that the person with dementia enjoyed, in line with the person-centred approach emphasized during the caregiver training (Study 1). Feedback from trainers and caregivers was generally very positive. Several authors have raised concerns regarding possible negative effects of involving caregivers to present an intervention for people with dementia (Small et al., 1997; Moniz-Cook, 2006), but the current results provide no evidence that the intervention had a negative effect on caregiver well-being. No caregiver indicated that presenting the activities was a serious burden for them and caregiver quality-of-life and burden remained at baseline levels. Further, caregivers' sense of competence in caring for their relative with dementia improved after the intervention (Study 2). In the absence of a no-treatment condition we are unable to ascertain that this improvement was a direct result of the intervention, but the improved sense of competence is in line with the positive feedback from caregivers. Although a small number of caregivers reported that the activities had a negative effect on their relative with dementia, the majority of caregivers reported of positive effects on the person with dementia and quality-of-life ratings of the person with dementia did not deteriorate after the activities (Study 1).

The direct costs of the intervention were considerably lower than the published costs of other interventions. Costs of multicomponent interventions presented by health care professionals reported by Graff et al. (2008) and Eloniemi-Sulkava et al. (2009) were €1183 (USD 1251) and €2923 (USD 3091), respectively, per dyad. In both interventions the intervention costs reflected primarily staff costs. The significant increase in one category of health service costs in the treatment group in Study 1 was not directly linked to the 


\section{Dementia}

intervention because the waiting list group did not show a similar increase after they had received the intervention. Closer inspection of the data showed that the increase in health service costs was due to a single person with dementia whose baseline costs were already high, suggesting ongoing health problems.

Both studies have limitations. The numbers of participants were modest in both studies, the research coordinator was not blind to the allocated condition in Study 1 and Study 2 did not have a control condition. Furthermore, in both studies the researchers had limited control over the selection of trainers and participants, the delivery of the intervention by trainers and caregivers and the completion of outcome measures. As a consequence, there was limited information on dementia severity and some missing data in outcome measures at follow-up. These limitations due to a lack of control are inevitable when attempting to implement interventions in real-life care settings and are among the important challenges for translation research (Teri et al., 2012). Further studies should evaluate the long-term implementation and sustainability of the intervention. The care and support organisations involved in the studies reported here were intending to continue presenting the training. Since the completion of our studies, several of the participating organisations have indeed approached the authors seeking further training opportunities. In addition, future research should evaluate in a randomised-controlled trial whether the intervention is effective in maintaining well-being and daily functioning in people with dementia and their family caregiver over longer periods of time and in postponing admission to residential care of the person with dementia. What the current study has shown is that involving trained nonprofessionals to present a multi-component intervention for caregivers and people with dementia can reduce the costs of the intervention and demand on health care services, which in turn can help to improve access to the intervention. 
Acknowledgements

We thank staff and volunteers from XXX who participated as trainers, and XXX at the Health Economics Research Unit of XXX who contributed to the economic analysis.

\section{Declaration of Conflicting Interests}

The authors declared no potential conflicts of interest with respect to the research, authorship, and/or publication of this article.

\section{Funding}

The authors disclosed receipt of the following financial support for the research, authorship, and/or publication of this article: This work was supported by XXX (Study 1) and XXX (Study 2).

\section{References}

Bellg A, Borrelli B, Resnick B, Hecht, J., Minicucci, D., Ory, M., ... Czajkowski, S. (2004). Enhancing treatment fidelity in health behavior change studies: Best practices and recommendations from the NIH behavior change consortium. Health Psychology, 23, $443-451$.

Chien, L., Chu, H., Guo, J. Liao, Y., Chang, L., Chen, C. \& Chou, K. (2011). Caregiver support groups in patients with dementia: a meta-analysis. Int J Geriatr Psychiatry, 26, 108998.

Curtis, L. (2013). Unit Costs of Health and Social Care 2013. Personal Social Services Research Unit. The University of Kent.

Dröes, R-M., Breebaart, E., Meiland, F., van Tilburg, W. \& Mellenbergh, G. (2004) Effect of Meeting Centres Support Program on feelings of competence of family carers and 
delay of institutionalization of people with dementia, Aging \& Mental Health,8:201-

211.

Eloniemi-Sulkava, U., Saarenheimo, M., Laakkonen, M., Pietila, M., Savikko, N., Kautiainen, H., Tilvis, R., \& Pitka, K. (2009). Family care as collaboration: Effectiveness of a multicomponent support program for elderly couples with dementia. Randomized controlled intervention study. Journal of the American Geriatric Society, 57, 22002208.

Gaugler, J., Yu, F., Krichbaum, K., \& Wyman, J. (2009). Predictors of nursing home admission for persons with dementia. Medical Care, 47, 191-198.

Gitlin, L., Winter, L., Vause Earland, T., Herge, E., Chernett, N., Piersol, C., \& Burke, J. (2009). The Tailored Activity Program to reduce behavioral symptoms in individuals with dementia: Feasibility, acceptability, and replication potential. The Gerontologist, 49, 428-439.

Gitlin, L., Marx, K., Stanley, I., \& Hodgson, N. (2015). Translating evidence-based dementia caregiving interventions into practice: state-of-the-science and next steps. The Gerontologist, 55, 210-226.

Graff, M., Adang, E., Vernooij-Dassen, M. , Dekker, J., Jonsson, L., Thijssen, M., Hoefnagels, W., \& Olde Rikkert, M. (2008). Community occupational therapy for older patients with dementia and their care givers: cost effectiveness study. British Medical Journal, 336, 134-138.

Jensen, M., Agbata, I., Canavan, M. \& McCarthy, G. (2014). Effectiveness of educational interventions for informal caregivers of individuals with dementia residing in the community: Systematic review and meta-analysis of randomised controlled trials. International Journal of Geriatric Psychiatry, 30, 130-143. 
Lawton, M., \& Brody, E. (1969). Assessment of older people: self-maintaining and instrumental activities of daily living. The Gerontologist, 9, 179-186.

Livingston, G., Johnston, K., Katona, C., Paton, J. \& Lyketsos, C. (2005). Systematic review of psychological approaches to the management of neuropsychiatric symptoms of dementia. American Journal of Psychiatry, 162, 1996-2021.

Logsdon R., Gibbons, L., McCurry, S., \& Teri, L (1999). Quality of life in Alzheimer's disease: patient and caregiver reports. Journal of Mental Health and Aging, 5, 21-32.

Milders, M., Bell, S., Lorimer, A., MacEwan, T. \& McBain, A. (2013). Cognitive stimulation by caregivers for people with dementia. Geriatric Nursing, 34, 267-273.

Mittelman, M., Roth, D., Coon, D. \& Haley, W. (2004). Sustained benefit of supportive intervention for depressive symptoms in caregivers of patients with Alzheimer's Disease. American Journal of Psychiatry, 161, 850-856.

Moniz-Cook E. (2006). Cognitive stimulation and dementia. Aging \& Mental Health, 10, 207210.

NHS Education for Scotland (2011). Mental Health in Scotland. A Guide to delivering evidencebased psychological therapies in Scotland. "The Matrix - 2011".

Onder, G., Zanetti, O., Giacobini, E., Frisoni, G. Bartorelli, L., Cardone, G., Lambertucci, P., Silveri, M. \& Bernabei, R. (2005). Reality orientation therapy combined with cholinesterase inhibitors in Alzheimer's disease: randomised controlled trial. British Journal of Psychiatry, 187, 450-455.

Olazarán, J., Reisberg, B., Clare, L., Peña-Casanova, J., del Ser, T,... Muñiz, R. (2010). Nonpharmacological therapies in Alzheimer's Disease: A systematic review of efficacy. Dementia and Geriatric Cognitive Disorders, 30, 161-178.

Prick, E., de Lange, J., Twisk, J. \& Pot, A. (2015). The effects of a multi-component dyadic intervention on the psychological distress of family caregivers providing care to people 
with dementia: a randomized controlled trial. International Psychogeriatrics, 27, 20132044

Quayhagen, M. \& Quayhagen, M. (2001). Testing a cognitive stimulation intervention for dementia caregiving dyads. Neuropsychological Rehabilitation, 11, 319-332.

Robinson, L., Iliffe, S., Brayne, C., Goodman, C. Rait, G., Manthorpe, J., Ashley, P., \& MonizCook, E. (2010). Primary care and dementia: 2. long-term care at home: psychosocial interventions, information provision, carer support and case management. International Journal of Geriatric Psychiatry, 25, 657-664.

Small G., Rabins, P., Barry P., Buckholtz, N., DeKosky, S., Ferris, S., ... Tune, L. (1997). Diagnosis and treatment of Alzheimer disease and related disorders: consensus statement of the American Association for Geriatric Psychiatry, the Alzheimer's Association and the American Geriatric Society. Journal of the American Medical Association, 278, 13631371.

Teri, L., Gibbons, L., McCurry, S., Logsdon, R., Buchner, D., Barlow, W., ... Larson, E. (2003). Exercise plus behavioral management in patients with Alzheimer disease: A randomized controlled trial. Journal of the American Medical Association, 290, 20152022.

Teri., L., McKenzie, G., Logsdon, R., McCurry, S., Bollin, S., Mead, J. \& Menne, H. (2012). Translation of two evidence-based programs for training families to improve care of persons with dementia. The Gerontologist, 52, 452-459.

Van't Leven, N., Prick, A., Groenewoud, J., Roelofs, P., de Lange, J. \& Pot, A. (2013). Dyadic interventions for community-dwelling people with dementia and their family caregivers: a systematic review. . International Psychogeriatrics, 25, 1581-1603. 
Vernooij-Dassen, A. Felling, E. Brummelkamp, M. Dautzenberg, G. van den Bosch, R. Grol. A short sense of competence questionnaire (SSCQ): measuring the caregiver's sense of competence. J Am Geriatr Soc 1999; 47: 256-7.

Ware, J., Kosinski, M., \& Keller, S. (1996). A 12 item short form health survey: construction of scales and preliminary tests of reliability and validity. Medical Care, 34, 220-233.

Wimo, A., Karlsson, G., Jönsson, B., \& Winblad, B. (1998). The Health Economics of dementia, Wiley's, London, United Kingdom.

Wimo, A. \& Prince M. (2010). World Alzheimer Report 2010. London: Alzheimer's Disease International.

Zarit, S., Orr, N. \& Zarit, J. (1985). The hidden victims of Alzheimer's disease: Families under stress. New York: New York University Press. 
Table 1.

Age and gender distribution of caregivers and people with dementia in the treatment and waiting list groups in Study 1.

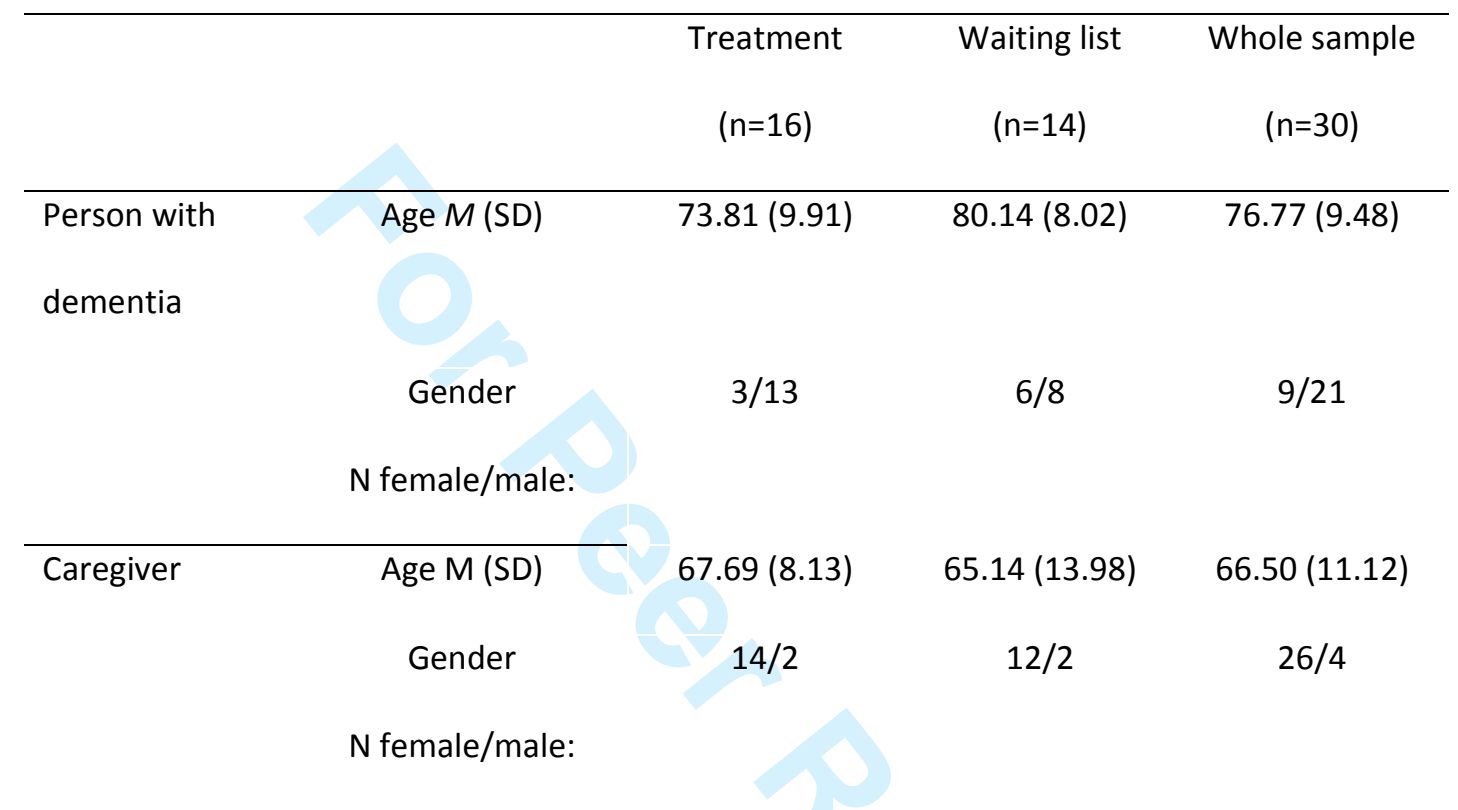


Table 2.

Well-being, daily functioning, caregiver burden and health service cost in the treatment and waiting list groups at baseline, 3-month and 6-month follow-up in study 1.

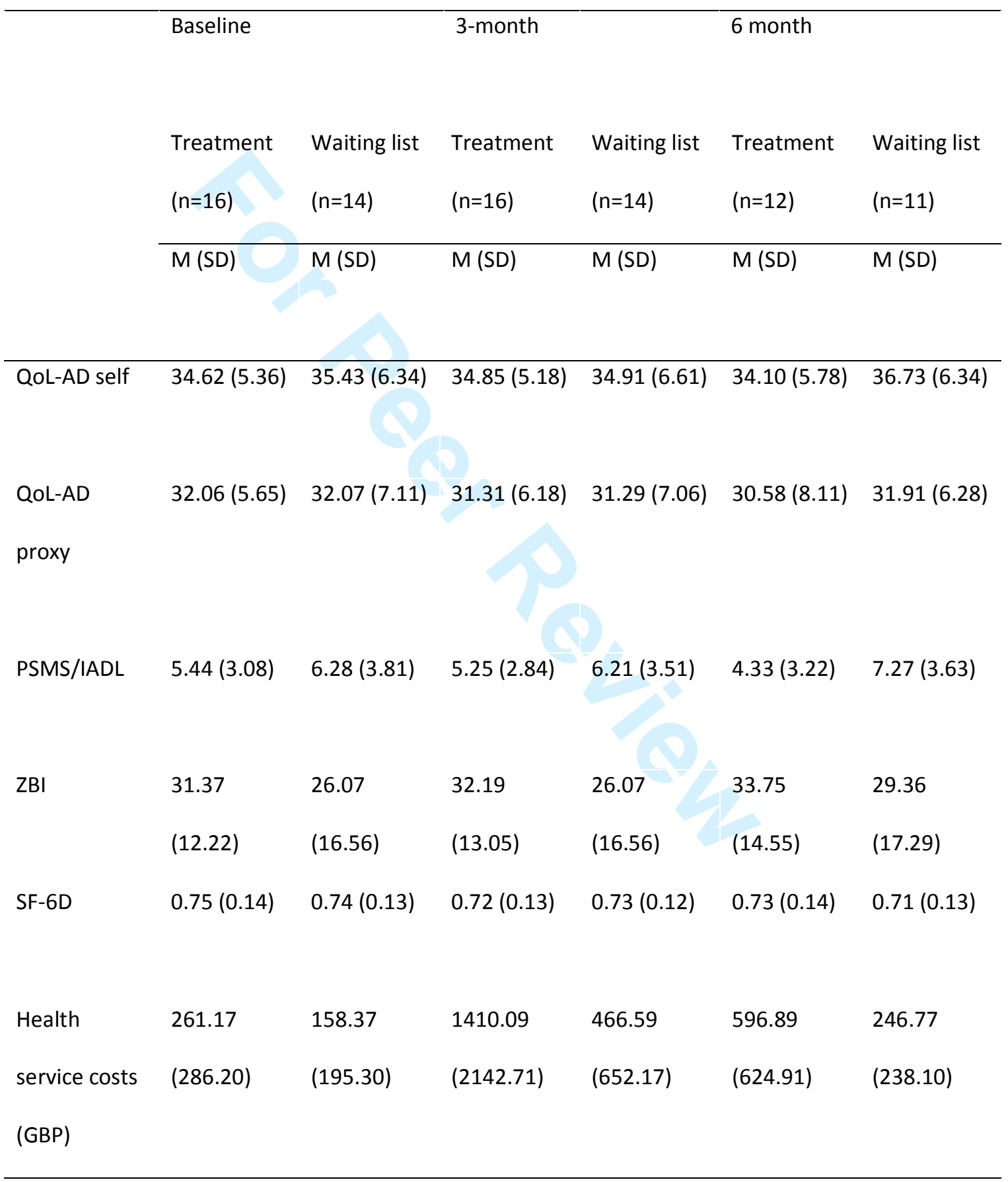


Table 3

Caregiver burden, well-being and sense of competence in caregivers at baseline and 3-month follow-up in Study 2.

\begin{tabular}{lll}
\hline & Baseline & 3-month \\
& $M(S D)$ & $M(S D)$ \\
ZBI & $34.17(13.19)$ & $34.31(12.68)$ \\
& & \\
& & \\
SF-6D & $0.68(0.12)$ & $0.67(0.13)$ \\
& & \\
Sense of competence & $23.13(5.01)$ & $24.26^{*}(4.99)$ \\
\hline${ }^{*} \boldsymbol{p}<.05$ & & \\
\hline
\end{tabular}

